\title{
BMJ Open How is poststroke fatigue understood by stroke survivors and carers? A thematic analysis of an online discussion forum.
}

\author{
Karen Thomas, ${ }^{1}$ Chloe Gamlin, ${ }^{1}$ Anna De Simoni, ${ }^{2}$ Ricky Mullis, ${ }^{1}$ Jonathan Mant ${ }^{1}$
}

To cite: Thomas K, Gamlin C, De Simoni A, et al. How is poststroke fatigue understood by stroke survivors and carers? A thematic analysis of an online discussion forum.. BMJ Open 2019:9:e028958. doi:10.1136/ bmjopen-2019-028958

- Prepublication history for this paper is available online. To view these files please visit the journal online (http://dx.doi. org/10.1136/bmjopen-2019028958).

Received 4 January 2019 Revised 12 June 2019 Accepted 13 June 2019
Check for updates

(C) Author(s) (or their employer(s)) 2019. Re-use permitted under CC BY-NC. No commercial re-use. See rights and permissions. Published by BMJ.

${ }^{1}$ Department of Public Health and Primary Care, University of Cambridge, Cambridge, UK

${ }^{2}$ Centre for Primary Care and

Public Health, Queen Mary

University of London, London, UK

Correspondence to

Karen Thomas;

kt439@medschl.cam.ac.uk

\section{ABSTRACT}

Objective To understand poststroke fatigue from the perspective of stroke survivors and caregivers expressed in an online discussion forum.

Design The search terms 'tiredness', 'fatigue', 'tired', 'weary' and 'weariness' were used to identify relevant posts. Thematic analysis performed by two independent researchers who coded all forum posts and identified pertinent themes. Posts were coded in relation to two research questions: (1) how is poststroke fatigue described? and (2) what coping strategies are suggested to target poststroke fatigue? Each theme was then summarised by a lead quotation in forum users' own words.

Setting UK-based web forum hosted by Stroke Association, TalkStroke. Archives from 2004 to 2011 were accessed. Participants 65 stroke survivors and caregivers (mean age 54 years, $61 \%$ female) contributed to 89 relevant posts that included a relevant search term. This included 38 stroke survivors, 23 individuals with family or carer role and 4 others unidentified.

Results Six themes were generated: (1) medicalisation of poststroke fatigue: 'a classic poststroke symptom', (2) a tiredness unique to stroke: 'a legacy of stroke', (3) normalisation and acceptance of poststroke fatigue: 'part and parcel of stroke', (4) fighting the fatigue: 'an unwelcome guest', (5) survivors' and caregivers' biological explanations: 'the brain healing' and (6) coping mechanisms: 'pace yourself'. Forum users also repeatedly commented that poststroke fatigue was 'not understood by the profession'.

Conclusion This is the first study to employ data from an online forum to characterise poststroke fatigue. Our data are considered naturalistic owing to the absence of a researcher guiding the discussion and thus generates useful insights for healthcare professionals. Findings suggest a requirement for consistent understanding and explanation to be provided by healthcare professionals. The beliefs outlined here highlight the gap between clinical and community knowledge. Further research to translate understanding of patient and carer perspective into improved management of poststroke fatigue is required.

\section{INTRODUCTION}

Every year in the UK, 110000 individuals experience a stroke. ${ }^{1}$ Prevalence of fatigue after stroke has been reported to be as high as
Strengths and limitations of this study

- Analysis of data from an online community enables naturalistic data collection without the potential bias associated with research interviews.

- This method of data collection employs direct analysis of posts written by stroke survivors and carers in the context of the forum, rather than in an 'unnatural' research setting.

- Using an online forum data set in this manner can complement traditional data collection techniques such as research interviews for qualitative research.

- Forum users may not be typical of the stroke community; they tend to be younger and have less severe strokes (though this can be off-set by caregivers' contributions).

$70 \%$, yet there is currently minimal evidence on which to base an effective management strategy. ${ }^{2-4}$ Among stroke survivors living in the community with fatigue, $43 \%$ report this need as unmet. ${ }^{5}$ Fatigue after stroke adversely affects survivors' quality of life, social participation, return to work and survival. ${ }^{26} 7$

Better understanding of poststroke fatigue (PSF) would enable healthcare professionals to identify patients with clinically significant fatigue who may benefit from further investigation and support. ${ }^{6}$ However, defining PSF is challenging due to its complex biopsychosocial elements and its "inherent subjectivity'. 8 This may be mitigated by incorporating an improved understanding of survivors' perceptions into a working definition of PSF for healthcare professionals. ${ }^{10}$

PSF varies in its clinical presentation, and the mechanisms that underlie it are poorly understood. $^{11}$ Some patients suffer from PSF for many years more than others with equivalent neurological damage. ${ }^{11}$ There is some evidence to suggest that the experience of PSF is associated with factors such as anxiety, reduced quality of life and physical 
activity. ${ }^{12}$ It has been proposed that PSF should be considered independently from other associated conditions such as depression, pain and sleep disorders. ${ }^{13}$ Although PSF is highly subjective in nature, there is limited published research exploring the patient narrative. ${ }^{11} \mathrm{~A}$ better understanding of the patient perspective within a biopsychosocial approach might facilitate a better understanding of the condition. ${ }^{14} 15$

Online forums provide a rich source of data from which subjective experiences of the issues pertinent to the population in question may be collected. ${ }^{16}$ Online communities increasingly provide a platform for patients and caregivers to seek information, support and discuss their conditions. ${ }^{17}$ The resulting data are generated from natural interactions between fellow forum users rather than from guided discussions with researchers. ${ }^{17}$ As such, analysis of online communities can uncover additional material in comparison to traditional qualitative methodologies. ${ }^{16}$

This study aimed to understand PSF by analysis of discussions in an online forum dedicated to stroke survivors and their caregivers. We asked two research questions: (1) how is PSF described? and (2) what coping strategies are suggested to target PSF?

\section{METHODS \\ Design}

This study employed thematic analysis of posts relating to PSF written by stroke survivors and caregivers on the archived TalkStroke Forum. The moderated forum comprises 22173 unique posts, 2583 usernames and was a UK-based online community hosted by the Stroke Association charity. We analysed archived forum posts including our primary search term 'tiredness' and related terms 'fatigue', 'tired', 'weary' and 'weariness' from a total of 71 TalkStroke forum participants, written by stroke survivors and their caregivers between 2004 and 2011. All demographic data were extracted through reading of subsequent posts by each user, and no information was taken from the individual registration process. ${ }^{17}$

\section{Patient and public involvement}

Following initial review of the literature, researcher KT visited a Cambridgeshire-based stroke group to discuss community-based patients understanding and experience of PSF. Initial research priorities were pitched to 18 individuals to decipher if the study research questions were thought to be important to the stroke survivor population. Individuals described the fatigue as overpowering and highlighted that when it hits, it is impossible to ignore. When asked about what hurdles they have faced following the stroke event, one individual stated that they felt fatigue was the root of their other concerns. It was clear that improving understanding and management for PSF should be a priority, and the current study will help address patients concerns.

\section{Ethics}

The Stroke Association gave researcher ADS access to the forum archives and permission for the data to be used for research purposes. It is important to note that use of internet data raises ethical questions including considerations of intrusiveness and perceptions of a forum as public or private. De Simoni and colleagues note that this specific analysis is classified as low intrusiveness, and due to the high number of users, participants were likely to view their posts as public. TalkStroke data were stored and accessed through the University of Cambridge Clinical School Secure Data Hosting Service. People signing up to TalkStroke agreed that their data were public on registering for the forum. To protect the identity and intellectual property of forum participants, we report fragments of responses and paraphrase longer discussion points.

\section{Data selection}

Two researchers KT and CG searched the forum archives for the following search terms: 'tiredness', 'tired', 'fatigue', 'weary' and 'weariness'. Repeated and irrelevant posts were removed, as agreed by KT and CG through use of two rounds of coding and discussion. Posts with only a cursory mention of tiredness/fatigue or fatigue in relation to depression, pain or sleep disorders were excluded, as they strayed from the focus on fatigue as a direct consequence of stroke. Posts written by individuals under the age of 18 years or by a parent figure describing their child were also removed since the focus of this study was adult stroke. This gave a preliminary data set of 104 posts written by 71 forum users. These posts were again screened by KT and CG to assess their relevance to the research questions and discussed until unanimous agreement was reached regarding the final data set, which comprised 89 posts written by 65 individuals.

\section{Data analysis}

After reading all posts in the final data set to ensure familiarisation with the content, two researchers (KT and CG) carried out inductive thematic analysis to generate and refine emerging issues, using methods described by Braun and Clarke. ${ }^{18}$ Posts were coded in order to answer the two research questions by identifying the defining characteristics of PSF and revealing coping strategies. The majority $(65 \%)$ of posts were coded by the first author (KT). Coding was performed independently for $30 \%$ of posts by the second author (CG). To ensure that a consistent, systematic coding style was used, both researchers coded five per cent of posts. Coding was discussed until agreement was reached for all posts to identify all pertinent key themes. These codes were aggregated into broader themes. During this process, both researchers revisited all extracts to ensure the suggested themes incorporated all data entries, and thus the six final themes truly represented the complete data set. The process for data selection and analysis is summarised in figure 1 . 


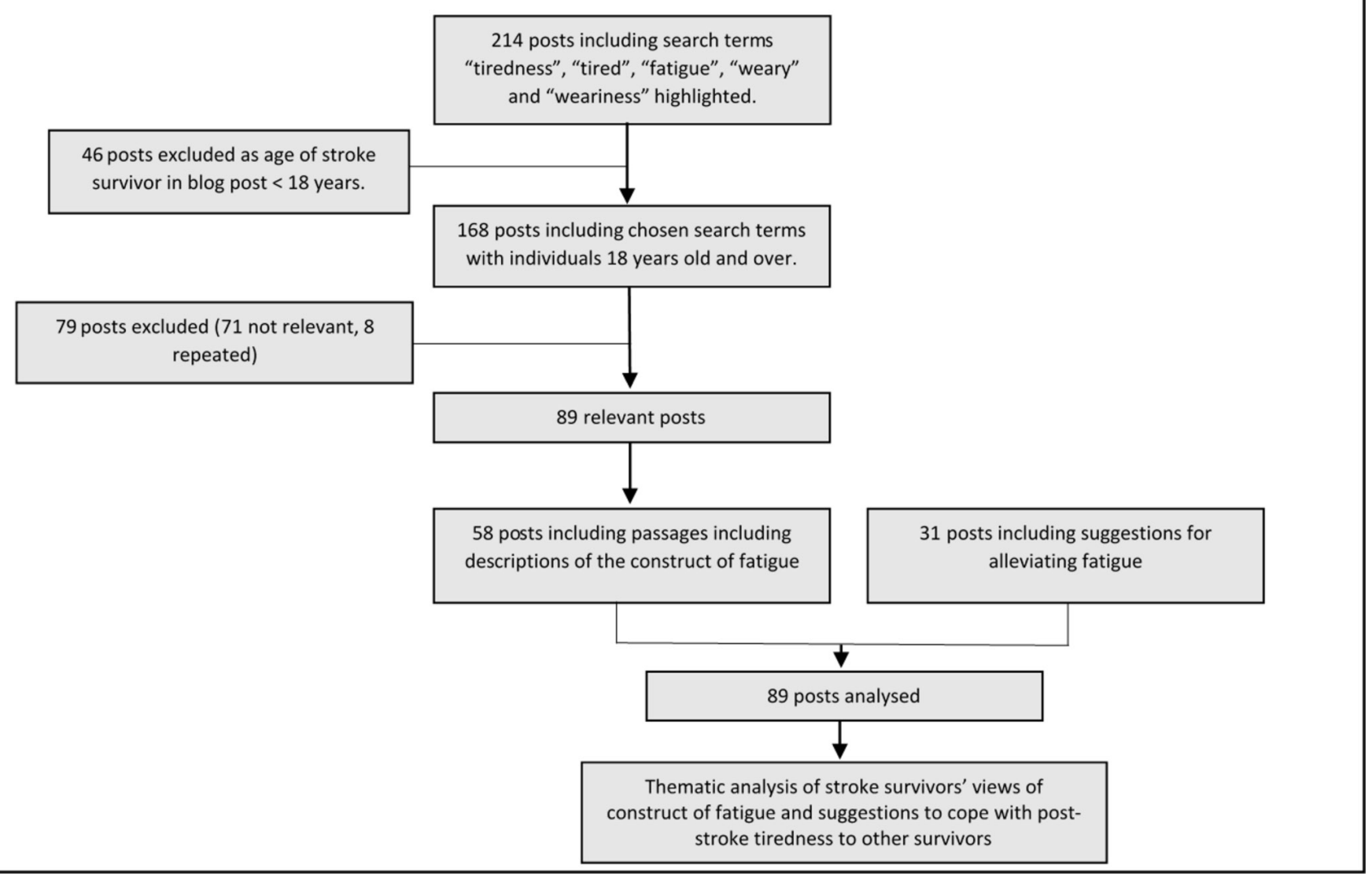

Figure 1 Flow chart of process for data selection and analysis.

\section{RESULTS}

\section{Participant characteristics}

Sixty-five individuals wrote 89 posts in the TalkStroke forum, which included the selected search terms. This included 38 stroke survivors, 23 individuals in a family or carer role and 4 others who were unidentified. The above demographics specifically refer to stroke survivors either posting about their own experience or being referred to by a carer figure using the forum.

\section{Themes}

We identified six themes representing a wide variety of understanding and approaches to PSF by stroke survivors and caregivers alike. Following data familiarisation, using the two research questions, 62 initial codes were created and discussed to enable collation into the final six themes. KT and CG identified codes emerging from survivor and carer posts to check for differences and due to no clear variance identified, the final six themes were created encompassing both experiential viewpoints.

\section{Medicalisation of PSF: 'a classic poststroke symptom'}

Forum participants employed language highly suggestive of medicalisation such as 'suffers with fatigue' (caregiver (CG) 4). Yet there was also much discussion about longterm effects of stroke as "not understood by the profession' (stroke survivor (SS) 37). One individual reported attending two outpatient appointments following discharge from the stroke ward in which his fatigue was never addressed. This is at odds with the strongly held idea by many stroke survivors on the forum that 'tiredness is very much a part of stroke symptoms... a classic poststroke symptom' (SS30). Notably, there is ample information about PSF available online and in booklet format from various charitable organisations such as The Stroke Association and Chest, Heart \& Stroke Scotland, but these recourses do not yet appear to be included in clinical practice, and were not mentioned in forum discussions.

\section{Tiredness like no other: 'a legacy of stroke'}

A number of forum users discussed the features of PSF itself. It was described as 'a fatigue like no other' (CG3) and a 'neurological tiredness' (SS6). There were multiple references to the idea that 'stroke can and does cause fatigue' (SS36) and that fatigue is 'a feature of our affliction' (SS9), and some took this further, characterising PSF as a distinct problem, 'a thing in itself, aside from chronic fatigue syndrome' (SS44). Furthermore, fatigue was repeatedly expressed by forum users as a 'legacy of stroke' (SS45) or a 'typical post-stroke legacy' (SS12), encapsulating survivors' experiences of a long-lasting fatigue directly linked to the stroke.

\section{Acceptance and normalisation of PSF: 'part and parcel'}

Often, stroke survivors asked other forum users 'is this tiredness normal?' (SS3), obtaining a plethora of affirmative responses. This can be summarised by the idea proposed by one participant that PSF 'is a guest you're 
stuck with, you've just got to learn to live with it' (SS12) and that 'the feelings are normal and all stroke survivors can relate to the tiredness' (SS29). Along with the reassurance that 'tiredness (fatigue) is very common post stroke' (CG16), forum posters acknowledged fatigue as an after effect of stroke. One post from a survivor held that 'tiredness is common and can last for years post stroke' (SS12). Normalisation was a recurring response to queries about PSF, demonstrated by the stroke survivor who wrote 'the exhaustion as other posts have said is normal' (SS5).

\section{Fighting PSF: 'unwelcome guest'}

In contrast, some TalkStroke forum users considered PSF to be 'one of those things we have to try and combat' (SS17). Forum users received advice from some individuals to 'not give into it' (CG8), to be 'continually fighting what's going on'(SS4) and to 'find your inner strength, [don't] let anything beat you' (CG2). Forum posts of a combative nature described the tiredness as 'annoying', emphasising that the 'point is not to give into it, [but] to find ways, little ways, to fight' (SS17). Despite this, such posts acknowledged that 'tiredness after stroke is very common' (SS20), much the same as the forum users accepting and normalising their PSF.

\section{Survivors' biological explanations and beliefs: 'brain healing'}

Most commonly, forum users attributed PSF to 'the brain healing' (SS17). We identified several variations on this theme: some stroke survivors and caregivers understood it as 'giving the brain the 'time off' it needs to start healing itself' (SS21), while others suggested that the 'body is working overtime trying to make sense of what has happened and heal as fast as it can' (CG16). There were also references to information about PSF provided by healthcare professionals. On one occasion, the phrase 'poststroke fatigue' was employed, where a relative explained he was told by a healthcare professional that his father in law 'may have post-stroke fatigue' (CG17). However, discussions usually centred around the explanation of symptoms given by healthcare professionals, that 'stroke causes fatigue' (SS12). Forum users sought to extend such explanations using metaphor for the disease process: 'the clock in your head... is going round at its own pace now' and expressed reverence for the brain, 'a strange thing' (CG9) they could not understand.

\section{How survivors cope with PSF: 'pace yourself'}

Forum posts discussing tiredness often mentioned or sought advice on how to cope with PSF. There was much discussion about involving healthcare professionals to assist with PSF management. Often, healthcare professionals were approached for support with the practicalities of returning to employment after stroke. For instance, one user reported that 'my GP doesn't really have any opinion on the tiredness (but is happy to keep signing me off from work)' (SS27). Another survivor was supported by forum users posting that just because your doctor cannot help [with the tiredness] it does not mean the be all and end all' (SS5). Forum users recommended simple practical solutions such as 'pacing yourself' (CG15) rather than approaching healthcare professionals for support in managing the fatigue itself. The online community suggested a plethora of lifestyle modifications to manage fatigue including 'learning to live within your new limitations and taking it easy when needed' (SS12).

For some stroke survivors, PSF represents an emotional struggle. One user described being pleased with his progress yet was annoyed at himself for concentrating on his 'physical recovery' without taking his 'mental recovery' (SS8) into account. Included in his 'mental recovery' was the idea of 'bouts of fatigue' that 'knocked [him] for six'. Yet for others, overcoming the fatigue was considered part of the physical recovery from stroke. One survivor reported that 'apart from tiredness and intermittent vertigo the physical effects of a stroke ... have thankfully passed' (SS14).

\section{Summary}

Table 1 includes fragments of quotes and paraphrasing of comments from the TalkStroke forum representative of our six themes

\section{DISCUSSION}

For stroke survivors and their caregivers, the forum provided legitimacy by acknowledging the existence of PSF, as well as offering management strategies based on lived experience. The homogeneity of forum users' interpretations of PSF is of particular note-the symptoms were repeatedly described as a 'legacy of stroke' (SS45), and the existence of PSF in this online community was rarely, if ever, questioned.

Forum users reported a number of conflicting approaches to managing fatigue with some patients being told by their healthcare providers to rest and others being given no information at all. Commonly included in such posts were quotes to the effect that PSF was "not understood by the medical profession' (SS37). As a result, stroke survivors and caregivers would seek out and offer their own, often metaphorical, explanations. Understanding and defining fatigue in clinical settings has provided a challenge for many decades due to the complex interaction of biological, psychosocial and psychological elements, which makes understanding and management difficult for clinicians and patients alike. ${ }^{19}$ The reported lack of medical consensus over approaches to PSF reflects an absence of a standardised approach to PSF in the medical setting. Ambiguity extends to understanding of how fatigue after stroke occurs and what factors influence its onset and longevity This also holds true in literature exploring fatigue management with Wu's 2015 Cochrane review being the only review to seek answers to whether any intervention reduces the proportion of people with fatigue, fatigue severity or both. Methodological quality was poor throughout, and no studies included primarily studied the effect on PSF. Treatment modalities included pharmaceutical 
Table 1 Themes based on exemplar quotes from the TalkStroke Forum archives

Themes Selected user quotes and paraphrased discussion points

Medicalisation of PSF A man replied that he suffered from tiredness. (CG6)

His consultant told him that tiredness is a major after effect of stroke. (SS9)

She described tiredness to another user as a classic post stroke symptom. (SS30)

A fatigue unique to When reflecting on PSF, a man commented that he felt everybody seemed to suffer from tiredness stroke following a stroke, further describing it as a silent disability that is not often talked about unless it is brought up in discussion. (SS8)

When replying to other forum users, one woman described PSF as a stroke legacy. (SS12)

One man told other users that he knew his stroke caused the fatigue he experienced after. (SS36)

Acceptance and One forum user used metaphor in a response to explain the length of time she believed fatigue may normalisation of PSF last for after a stroke, telling the user that fatigue is a guest you're stuck with and explaining that acceptance is important to be able to learn to live with it. (SS12)

One man responded to a concerned forum user to assure them that their feelings were normal and all stroke survivors could relate to post stroke tiredness. (SS29)

One stroke survivors husband reflected that his wife had learnt what the signs of tiredness were for her personally, and understood that they signal a need to rest. (CG5)

Fighting PSF

One male user highlighted that tiredness was a problem for him and he dealt with it by pushing onwards to fight the tiredness by keeping mentally and physically busy.

(SS12)

A woman replied that it was important to not give into the tiredness and to find strategies to combat it. (CG15)

By relateing her tiredness to the brain injury, one user responded that it can be overcome and the brain can outwit the need for sleep. (SS9)

$\begin{array}{ll}\text { Survivors' biological } & \text { One caregiver made sense of the tiredness by considering the tiredness as result of the brain damage } \\ \text { explanations and } & \text { caused by a stroke event. (CG9) } \\ \text { beliefs } & \text { A forum user wrote an affirmative response that the tiredness he often experienced was due to the } \\ \text { brain healing. (SS17) } & \text { A woman drew on information she had been given by a medical professional that PSF occurs as a } \\ & \text { result of the brain being tired. (SS18) } \\ & \text { One woman personified her brain, responding by explaining that if the brain does not get tired, it will } \\ \text { not have the rest time necessary to heal. (SS26) }\end{array}$

How survivors cope with PSF

\section{(A) Involving healthcare professionals}

One man told other users that his consultant had signed him off work as unfit for the forseeable future. (SS9)

Another forum user was told by her GP that tiredness was something she would have to get used to and it would improve with time. She reflected on this, writing that she needed to accept it was a part her now. (SS59)

(B) Her husband had been written off by therapists. She elaborated on this by saying they still said he had reached his full potential even after significant time has passed. (CG17)

(C) Lifestyle changes

One forum user advised others that they needed to learn to live within their poststroke limitations and take it easy when needed. (SS39)

A caregiver advised stroke survivor forum users that it was important to make plans to occupy their time, however to have a rest period if the tiredness became overwhelming. (CG24)

(D) Mental versus physical recovery

One forum user replied that he experienced bouts of fatigue. When describing how it felt, he used a figure of speech, saying that it knocked him for six. (SS14)

A daughter responded that the tiredness was still a big problem for her mum but she reflected on the importance of her mum understanding her own capabilities to know what is and isn't manageable. (CG13)

One man voiced frustration at concentrating on his physical recovery after the stroke more than his mental recovery when discussing his fatigue. (SS14)

CG, caregiver; PSF, poststroke fatigue; SS, stroke survivor.

options such as fluoxetine, enerion and citicoline. Non-pharmaceutical options were a fatigue education programme, a mindfulness-based stress reduction programme and continuous positive airway pressure. ${ }^{20}$

\section{Comparison with other literature}

PSF is reported as one of the largest unmet needs in stroke survivors. ${ }^{5}$ Despite this, fatigue is only recently starting to be included within prominent clinical guidelines. ${ }^{21-24}$ 
This is largely due to the lack of high-quality studies and methodological variation evident in the PSF literature. ${ }^{25}$ In a report by the European Stroke Organisation on evidence-based stroke rehabilitation, although several topics are discussed, fatigue is noticeably missing. ${ }^{23}$ The absence of guidance for clinicians working with this population is reflected in the absence of a standardised approach as was apparent in the online forum posts.

Analysis of online community content is arguably an underused research design, despite being found to potentially offer additional insight to traditional interviews. ${ }^{16}$ Other research using the TalkStroke forum archives have been found to complement evidence collected employing other research designs. Balasooriya-Smeekens et $a l \mathrm{~s}^{26}$ study found that residual impairments, including fatigue, affected stroke survivors' return to work. Forum users also discussed a multitude of other difficulties stemming from fatigue including feeling misunderstood and suffering from an 'invisible disability'. ${ }^{26}$ Our analysis found forum users referred to fatigue in this way. Balasooriya-Smeekens et al's study also alluded to the frequency of individuals experiencing fatigue after a stroke event, with over half of the forum users posting about occupation difficulties also pinpointing fatigue as an important factor. Recent research has examined factors associated with PSF and found reduced independence in activities of daily living and higher anxiety levels had a direct association with level of fatigue. ${ }^{27}$

\section{Strengths and limitations}

This paper is the first to explore PSF from the perspective of stroke survivors and caregivers through an online forum. Previous qualitative studies have found online forums to constitute a rich and important data source, where patient perspectives are given in open discussion in the absence of a researcher. ${ }^{16}$ As such, the insights into PSF gathered in our study provide a useful and valid contribution to the understanding of this common and debilitating problem.

A further strength of this study is that it employed a 'naturalistic' data collection methodology. ${ }^{16}$ Using an archived online forum means our data were created from natural interactions between fellow forum users, rather than from predefined or guided discussions with researchers, thus removing participant bias towards the research agenda. ${ }^{21}$ Therefore, we believe that this approach has enabled us to best elicit understandings and approaches to PSF from the perspective of stroke survivors and their caregivers.

A potential limitation of this study is that forum users may not be typical of all stroke survivors. Participants tend to be younger and less severely affected by stroke. ${ }^{1721} \mathrm{It}$ is possible that the constructs of PSF derived from our analysis of the forum posts are only representative of the beliefs of a self-selecting group of forum users. Forum users may also not represent all social classes, as De Simoni and colleagues ${ }^{17}$ highlighted by identifying over half of posters as holding professional occupations. However, this information was only gathered from a minority of users so should be considered with caution. Furthermore, the data collected were from archives dated before 2012. Qualitative research has been published since this date, and so this may not represent current understanding of PSF. This is, however, the most recent study investigating the topic within an online community.

By using a naturalistic data collection method, we were unable to identify the underlying cause of the fatigue that participants were discussing on the forum. Fatigue has been approached in this paper from a lay understanding, rather than from a 'medical model'. There is a chance we were not able to exclude all forum posters under the age of 18 years due to several participants having unknown age. The search terms utilised could also present a limitation, as Boolean operators were not used. Further qualitative research could incorporate a wider range of terms within the search strategy.

\section{Implications}

The array of conceptualisation and approaches to managing PSF identified in this analysis highlights the need for better evidence on how to optimise the recovery process for stroke survivors with fatigue and their caregivers. Our data suggest that some patients who had a stroke use the online community of stroke survivors and caregivers to provide informal explanations and reassurance. The construct of PSF as a 'tiredness like no other' (SS6) that 'everybody seems to suffer with' (SS24) suggests a requirement for a consistent understanding and explanation to be provided by healthcare professionals, reducing the reliance of sufferers to seek informal explanations and reassurance from within the stroke community. Capturing the most prevalent beliefs, largely that fatigue is 'due to the brain healing' (SS17), enables assessment of the gap between clinical and community knowledge informing the education required to better inform survivors. Better understanding by healthcare professionals of these lay beliefs may help them support their patients.

Acknowledgements We would like to thank the Stroke Association for providing us with access to forum archives and Cambridge Stroke Group for allowing KT to conduct a Patient and Public Involvement (PPI) group prior to study commencement.

Contributors ADS and KT contributed to the initial design of the study. KT and CG conducted data analysis and theme creation. KT and CG wrote the initial manuscript. ADS, JM and RM reviewed and contributed to the final manuscript.

Funding This study was funded by the Malati Kanbur Studentship fund kindly donated by Ravi Kanbur and Margaret Grieco to Lucy Cavendish College, constituent college of the University of Cambridge. JM is a National Institute for Health Research Senior Investigator.

Disclaimer Views expressed in this written publication are those of the authors and not necessarily those of the funders.

Competing interests None declared.

Patient consent for publication Not required.

Provenance and peer review Not commissioned; externally peer reviewed. Data sharing statement № additional data are available.

Open access This is an open access article distributed in accordance with the Creative Commons Attribution Non Commercial (CC BY-NC 4.0) license, which 
permits others to distribute, remix, adapt, build upon this work non-commercially, and license their derivative works on different terms, provided the original work is properly cited, appropriate credit is given, any changes made indicated, and the use is non-commercial. See: http://creativecommons.org/licenses/by-nc/4.0/.

\section{REFERENCES}

1. National Institute for Health and Care Excellence. Stroke and transient ischaemic attack in over 16s: diagnosis and initial management Clinical Guideline [CG68].

2. Glader EL, Stegmayr B, Asplund K. Poststroke fatigue: a 2-year follow-up study of stroke patients in Sweden. Stroke 2002;33:1327-33.

3. Leegaard OF. Diffuse cerebral symptoms in convalescents from cerebral infarction and myocardial infarction. Acta Neurol Scand 1983;67:348-55.

4. Wu S, Kutlubaev MA, Chun H-YY, et al. Interventions for post-stroke fatigue. Cochrane Database Syst Rev 2015;22:CD007030.

5. McKevitt C, Fudge N, Redfern J, et al. Self-reported long-term needs after stroke. Stroke 2011;42:1398-403.

6. De Groot MH, Phillips SJ, Eskes GA. Fatigue associated with stroke and other neurologic conditions: Implications for stroke rehabilitation. Arch Phys Med Rehabil 2003;84:1714-20.

7. Naess $\mathrm{H}$, Lunde L, Brogger J, et al. Fatigue among stroke patients on long-term follow-up. The Bergen Stroke Study. J Neurol Sci 2012;312:138-41.

8. Duncan F, Lewis SJ, Greig CA, et al. Exploratory longitudinal cohort study of associations of fatigue after stroke. Stroke 2015;46:1052-8.

9. Aaronson LS, Teel CS, Cassmeyer V, et al. Defining and measuring fatigue. Image 1999;31:45-50.

10. Aaronson LS, Teel CS, Cassmeyer V, et al. Defining and measuring fatigue. Image J Nurs Sch 1999;31:865-9.

11. Eilertsen G, Ormstad H, Kirkevold M. Experiences of poststroke fatigue: qualitative meta-synthesis. J Adv Nurs 2013;69:514-25

12. Ponchel $\mathrm{A}$, Bombois $\mathrm{S}$, Bordet $\mathrm{R}$, et al. Factors associated with poststroke fatigue: a systematic review. Stroke Res Treat 2015;2015:1-11.

13. Kuppuswamy A, Rothwell J, Ward N. A model of poststroke fatigue based on sensorimotor deficits. Curr Opin Neurol 2015;28:582-6.
14. Ingles JL, Eskes GA, Phillips SJ. Fatigue after stroke. Arch Phys Med Rehabil 1999;80:173-8.

15. Ormstad $\mathrm{H}$, Eilertsen $\mathrm{G}$. A biopsychosocial model of fatigue and depression following stroke. Med Hypotheses 2015;85:835-41.

16. Jamison J, Sutton S, Mant J, et al. Online stroke forum as source of data for qualitative research: insights from a comparison with patients' interviews. BMJ Open 2018;8:e020133.

17. De Simoni A, Shanks A, Balasooriya-Smeekens C, et al. Stroke survivors and their families receive information and support on an individual basis from an online forum: descriptive analysis of a population of 2348 patients and qualitative study of a sample of participants. BMJ Open 2016;6:e010501.

18. Braun V, Clarke V. Using thematic analysis in psychology. Qual Res Psychol 2006;3:77-101.

19. Aaronson LS, Teel CS, Cassmeyer V, et al. Defining and measuring fatigue. Image J Nurs Sch 1999;31:45-50.

20. Wu S, Kutlubaev MA, Chun H-YY, et al. Interventions for post-stroke fatigue. Cochrane Database Syst Rev 2015;22:Art. No.: CD007030.

21. National Institute for Health and Care Excellence. Stroke rehabilitation: long-term rehabilitation after stroke (clinical guideline CG162), 2013.

22. Quinn TJ, Paolucci S, Sunnerhagen KS, et al. Evidence-based stroke r-ehabilitation: an expanded guidance document from the european stroke organisation (ESO) guidelines for management of ischaemic stroke and transient ischaemic attack 2008. J Rehabil Med 2009;41:99-111.

23. Royal College of Physicians. National clinical guideline for stroke: fourth edition, 2012. ISBN 978-1-86016-492-7.28.

24. Hinkle JL, Becker KJ, Kim JS, et al. Poststroke fatigue: emerging evidence and approaches to management: a scientific statement for healthcare professionals from the American Heart Association. Stroke 2017:48:e159-e170.

25. McGeough E, Pollock A, Smith LN, et al. Interventions for poststroke fatigue. Cochrane Database Syst Rev 2017;3:CD007030.

26. Balasooriya-Smeekens $\mathrm{C}$, Bateman A, Mant J, et al. Barriers and facilitators to staying in work after stroke: insight from an online forum. BMJ Open 2016;6:e009974.

27. Hawkins L, Lincoln NB, Sprigg N, et al. The Nottingham Fatigue After Stroke (NotFAST) study: results from follow-up six months after stroke. Top Stroke Rehabil 2017;24:592-6. 